\title{
«Alzheimer können wir nicht heilen - aber wir können vorbeugen»
}

\section{Daniel Lüthi}

Freier Journalist und Fotograf, Medientrainer, Bern

Bei der Lektüre seines Lebenslaufes fällt sofort auf: Dieser Altersmediziner hat seine Doktorarbeit über Embryonen geschrieben. Nicht deshalb, weil er sich zuerst bewusst mit dem Beginn des Lebens und dann mit dessen Ende habe befassen wollen, sagt er, «sondern weil ich Horror davor hatte, mich für eine Datenanalyse in einem Archiv zu verschanzen. Ich wollte eine experimentelle Doktorarbeit machen.»

\section{Breit arbeiten}

Konkret: Reto W. Kressig wollte schon damals forschen - und mit seinen Resultaten etwas bewirken. Da- mals untersuchte er die toxische Wirkung von Vitamin A bei Hühner-Embryonen mit dem Ziel, Erkenntnisse für die Behandlung von Psoriasis zu gewinnen. «Die Embryonen waren eher ein Zufall, wie ihn das Leben manchmal halt so schreibt. Mein Ziel war es, herauszufinden, ob ich labortauglich bin", kommentiert Kressig im Rückblick, «aber ich wusste bald, dass es das später mal nicht sein würde.»

Eigentlich hatte er im Sinn, Hausarzt zu werden, «deshalb brauchte ich einen gut gefüllten internistischen Rucksack. In der Inneren Medizin fand ich alles spannend, aber es war mir dann zu eng, mich auf ein einzelnes Organ zu spezialisieren. Ich wollte breit arbeiten

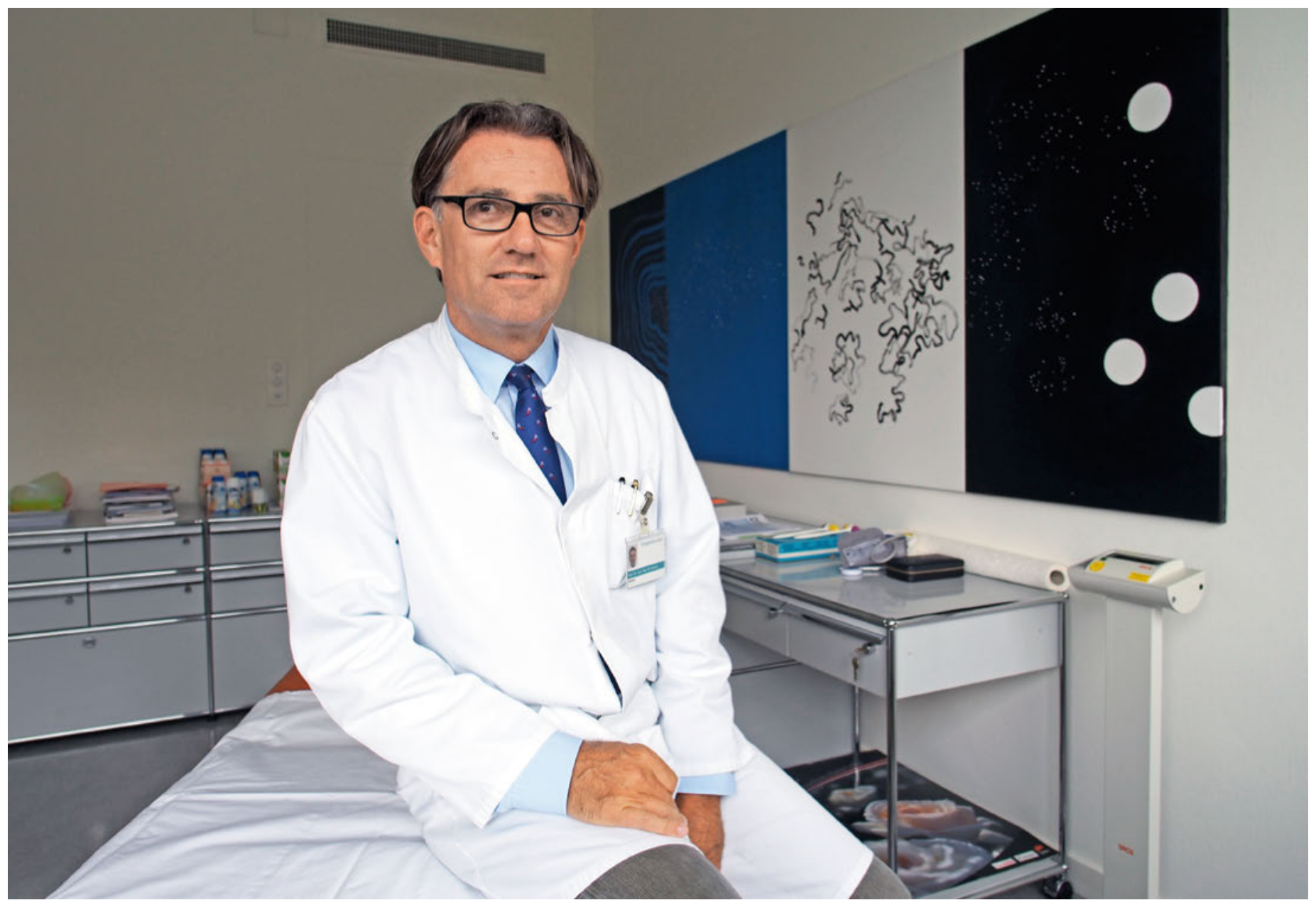


und doch Spezialist sein. In der Geriatrie des Bürgerspitals St. Gallen wusste ich: Das ist es!» Die Altersmedizin biete einen spannenden Mix aus medizinischer Komplexität und menschlich-sozialen Komponenten. Schon damals sei es darum gegangen, Prioritäten zu setzen. «Da gab es ältere Patienten, die 20 verschiedene Medikamente pro Tag schluckten. Wir mussten beurteilen, was wirklich nötig, und was möglicherweise sogar schädlich ist.»

\section{Fehlende Grundlagen}

Bleiben wir einen Moment beim Thema «Medikamente»: «Ja, die gehören auch dazu», sagt Kressig, gleichzeitig legt er seinen Finger aber auf einen wunden Punkt: «In meinem Spezialgebiet bin ich mit Medikamenten konfrontiert, die meist an jungen Leuten entwickelt und getestet worden sind, die wir Ärzte dann aber bei polymorbiden, alten Menschen anwenden sollen. Das ist etwas, was ich der Industrie und der Zulassungsbehörde vorwerfe: dass sie uns in diesem Bereich bewusst auf eine Patientengruppe extrapolieren lassen, die wissenschaftlich nicht untersucht worden ist. Mit Medikamentenstudien wird also für polymorbide Hochaltrige keine oder wenig Evidenz kreiert.» Ein gewisses Verständnis habe er dafür «aus ganz pragmatischen Gründen» schon, räumt Kressig ein: «Wenn ein Molekül entwickelt wird, ist halt viel Geld im Spiel. Dass man unter diesem Vorzeichen nicht gerade dort arbeitet, wo es methodologisch am schwierigsten ist, kann ich nachvollziehen.»

Aber: «Dem geschilderten Missstand könnten die Gesundheitsbehörden mit einer flächendeckenden Versorgungsforschung entgegenwirken. Leider haben wir keine Registerdaten. Wir würden sie dringend benötigen, aber dafür werden kaum Mittel gesprochen.» Gerade bei der Entwicklung der nationalen Demenz-Strategie habe er sich dafür eingesetzt, dass eine solche Bestandsaufnahme gemacht werde. «Bezüglich Demenz haben wir in der Schweiz keine fundierten Zahlen - wir müssen extrapolieren, mutmassen.»

\section{Vieles liegt im Dunkeln}

Wir sind im Zentrum des Wirkens von Reto W. Kressig angelangt: Demenz. Wir wissen, dass die absoluten Zahlen zunehmen, weil die Bevölkerung im Schnitt immer älter wird. «Aber die Inzidenz hat abgenommen. Das hat zu tun mit einem gesünderen Lebensstil und mit deutlichen Fortschritten bei der Behandlung von vaskulären Risikofaktoren wie Bluthochdruck», kommentiert Kressig und kommt jetzt so richtig in

\section{Reto W. Kressig}

Prof. Dr. med. Reto W. Kressig wurde 1960 in Zürich geboren. Er studierte Medizin an der Universität Zürich und doktorierte 1988 in Embryologie. Seine Assistenzzeit absolvierte er unter anderem in Brugg und in St. Gallen. 1994 erwarb er den Facharzttitel in Innerer Medizin, 1999 jenen in Geriatrie. Von 1994 bis 2006 arbeitete er am Universitätsspital Genf in verschiedenen klinischen Bereichen der Geriatrie,

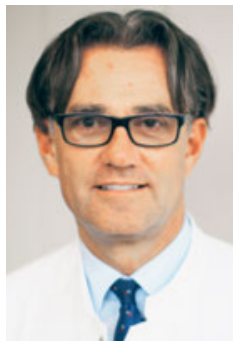
1998 bis 2000 forschte er zum Thema in Atlanta in den USA. Seit 2006 ist er in Basel Extraordinarius und Chefarzt für Geriatrie. Zurzeit vereinigt er in enger Kooperation mit dem Universitätsspital und den Universitären Psychiatrischen Kliniken die entsprechenden altersmedizinischen Abteilungen unter einem Dach. Das Felix Platter-Spital verschwindet, in Basel entsteht - auch als Gebäude - eine neue Universitäre Altersmedizin. Reto W. Kressig lebt mit seinem Partner in Basel.

Fahrt. Das heisst: Er spricht lange und druckreif, doziert bisweilen, bleibt aber immer auf Augenhöhe seines Gegenübers. «In der Schweiz gehen wir von rund 130000 Demenzkranken aus. Davon sind wohl nur etwa die Hälfte als solche diagnostiziert. Bei vielen gibt es weder Abklärung, Diagnose noch Therapie.» Ist eine klare Diagnose denn überhaupt möglich? Oder sind die Grenzen zwischen gesund und krank in diesem Bereich fliessend? «Wenn man's richtig macht, am besten in einer Memory-Klinik, kann man eine verlässliche Diagnose stellen», sagt Kressig. «Eine Hirnleistung, die sich abbaut, können wir messen.»

Es sei noch kein Alarmzeichen, wenn einem mal ein Name nicht in den Sinn komme oder man plötzlich nicht mehr wisse, was man gerade habe erledigen wollen. «Erst wenn jemand wiederholt seinen Alltag nicht mehr allein bewältigen kann oder wenn er Dinge ver-

\section{«Ein Sturz ist bei einem alten Menschen} oft der Beginn einer Abwärtsspirale.»

gisst, die ihm ganz wichtig sind, müssen wir von einer ernsthaften Störung der Hirnleistung ausgehen.» Kressig wehrt sich nicht dagegen, Themen immer wieder auch an sich selber zu spiegeln. Er selber könne sich gut an Gesichter erinnern, antwortet er auf die Frage nach der Vergesslichkeit, «aber Namen blende ich aus Selbstschutz oft aus».

Die häufigste Ursache von Demenz sei die AlzheimerKrankheit, erklärt der Geriatrie-Professor. «AlzheimerAblagerungen im Hirn in vivo nachzuweisen, ist möglich, der Aufwand aber ist enorm." Vor allem aber habe es im Moment keine therapeutischen Konsequenzen. «Mit Impfstoffen könnten wir diese Amyloid-Ablagerungen sogar wegbringen. Bloss: Die bisher verfüg- 
baren Studien haben gezeigt, dass sich die Hirnleistung dadurch nicht verbessert.» Zusammengefasst: "Alzheimer können wir nicht heilen - aber wir können vorbeugen.» Und hier gibt es eine weitere Entdeckung im Lebenslauf dieses Professors: den Zusammenhang zwischen dem Thema seiner Habilitation und seinem Schwerpunkt-Thema Demenz.

\section{Der Gang und das Gehirn}

Kressig hat habilitiert mit Untersuchungen im Bereich der Mobilität von alten Menschen. Konkret hat er Senioren über einen Teppich mit Sensoren gehen lassen und ihren Gang studiert, dessen Regelmässigkeit und Konstanz analysiert. «Früher haben Wissenschafter hier nur beobachtet. Wir haben gemessen und bewiesen.» Zum Beispiel: dass das Sturzrisiko bereits bei einem von blossem Auge nicht erkennbaren unregelmässigen Gang grösser wird. "Und ein Sturz ist bei einem alten Menschen oft der Beginn einer Abwärtsspirale.» Eine weitere Erkenntnis: «Wenn wir das Gehen mit kognitiven Aufgaben verbinden, und das Sturz-Risiko grösser wird, können wir auch von einem höheren Demenz-Risiko ausgehen.» Früher sei bei Demenzkranken lediglich der Zusammenhang hergestellt worden, dass etwas - zum Beispiel ein neuer Teppich in der Wohnung - «vergessen» und damit zur Stolperfalle wurde. «Heute wissen wir, dass unsere Gang-Motorik direkt mit unserer Hirnleistung zusammenhängt.» Rhythmik, Tanzen zu Musik seien eine gute DemenzPrävention. «Mit einer physischen Intervention können wir die Hirnleistung verbessern" - sagt einer, der eigentlich mal hätte Pianist und Organist werden wollen und dem Musik heute noch viel bedeutet. «Studien haben überdies gezeigt, dass das Spielen eines Instrumentes das Demenz-Risiko massiv reduzieren kann. Das hat mit einer zusätzlichen Aktivierung unseres Frontalhirns zu tun.» Womit wir bei der Evolution und bei einer weiteren zentralen Theorie von Reto W. Kressig angelangt sind: «Die Entwicklung des Frontalhirns - und damit die Entwicklung des Menschen hatte ihren Anfang, als der Mensch zu jagen und Fleisch zu essen begann.»

\section{Fleisch als Protein-Lieferant}

Auch dies eine Entdeckung: Wer im Internet zu diesem Mediziner recherchiert, findet einen Artikel von «Proviande», der Branchenorganisation der Schweizer Fleischwirtschaft. Titel: Die Rolle von Fleisch in der Seniorenernährung. Ein Zitat daraus: «Muskel kann auch im hohen Alter neu gebildet werden, wenn die Proteinzufuhr genügend ist.» Natürlich gebe es Eiweiss auch in Milchprodukten oder Tofu, sagt Kressig - bloss: «Wenn Sie wissen, dass wir im Alter pro Tag rund 100 Gramm Protein benötigen, wissen Sie auch, dass Sie gar nie so viel Soja essen können, um diesen Bedarf zu decken.» Und konkret darauf angesprochen stellt er fest: "Aus der Sicht eines Altersmediziners machen mir die zunehmenden Vegetarier und Veganer Sorgen. Eine junge Veganerin zum Beispiel geht ein Risiko ein dass ihr Kind mit Missbildungen auf die Welt kommt.» Wir sind wieder beim Beginn eines Lebens angelangt, und wollen den Kreis zur letzten Phase schliessen. «Kontrollverlust ist für mich keine Horrorvorstellung», sagt Reto W. Kressig. Immer wieder sehe er, wie viel Lebensqualität auch bei dementen Menschen möglich sei. «Das hat natürlich auch mit dem Setting zu tun.» Er selber habe für den Fall, dass ihn die Krankheit treffen würde, nicht vorgesorgt. "Ich weiss, dass man in den meisten Fällen noch Zeit hat, um Dinge zu regeln. Und dass ich in den Institutionen um mich herum gut aufgehoben wäre.» Im Übrigen berge das

\section{«Kontrollverlust ist für mich keine Horrorvorstellung."}

Altwerden auch Chancen: «Wir dürfen unsere eigenen Verlust-Vorstellungen nicht direkt auf ältere Menschen projizieren. Ich bin immer wieder beeindruckt davon, wie anpassungsfähig der Mensch ist. Und ich sehe regelmässig demente, kranke, behinderte Menschen, die in einer guten Stimmung sind.»

Ein ganzheitlicher Ansatz scheint dazu beizutragen. In Basel ist dieser zurzeit augenfällig: Eine riesige Baustelle erinnert daran, dass hier ein neues Universitäres Zentrum für Altersmedizin am Entstehen ist. Die frühere Akutgeriatrische Klinik des Universitätsspitals und das Felix Platter-Spital werden zusammengeführt, Reto W. Kressig ist ab 1. Januar Ärztlicher Direktor des neuen Hauses. Dies freut ihn besonders: «Wir wagen es, nebst einem modernen altersmedizinischen Akutspital und der Rehabilitation auch die Alterspsychiatrie unter das gleiche Dach zu nehmen. Das wird die Wege verkürzen, also vor allem unseren Patientinnen und Patienten dienen.»

Das werden sie spüren. Auch wenn sie nicht mehr viel wissen und merken.

Bildnachweis Fotos Daniel Lüthi

Die nächste Begegnung mit ...

Im November porträtiert Daniel Lüthi Prof. Hansjakob Furrer, Direktor und Chefarzt der Universitätsklinik für Infektiologie am Inselspital Bern. 